ARTÍCULOS ORIGINALES Rev Chil Salud Pública 2014; Vol 18 (3): 257-262

\section{PREVALENCIA DE EDENTULISMO PARCIAL Y TOTAL EN ISLAS BUTACHAUQUES Y TAC, QUEMCHI, 2013 *}

\author{
Prevalence of Partial and total eDentulism in butachauQues \\ AND TAC ISLANDS, QUEMCHI, (CHILE) 2013
}

Víctor CORTÉs

Felipe Carrasco

Alumno Interno Escuela de Odontología Facultad de Medicina Universidad Austral de Chile

vhcy.87@gmail.com

\section{Carolina Vergara}

Docente Adjunto Escuela Odontología e Instituto de Salud Pública

Facultad de Medicina Universidad Austral de Chile

\section{RESUMEN}

Objetivo: Determinar la prevalencia de edentulismo y clasificación topográfica de Kennedy en población mayor de 20 años de islas Butachauques y Tac.

Material y Método: Estudio observacional descriptivo. Se incluyeron sujetos mayores de 20 años en los servicios de salud de islas Butachanques y Tac durante los meses de agosto y septiembre de 2013. Se solicitó a cada sujeto de investigación la firma de un consentimiento informado. La información recopilada fue ingresada a una planilla de registro de datos diseñada para la investigación. Las variables utilizadas fueron género, edad, cantidad de dientes presentes, portador de prótesis y tipo de edentulismo según clasificación topográfica de Kennedy, para luego realizarse un análisis de estadistica descriptiva mediante SPSS 18 (IBM® SPSS® software).

Resultados: Fueron evaluados 88 sujetos, de los cuales un $39.7 \%$ hombres y un $60.3 \%$ mujeres. Un $89.7 \%$ de los sujetos presentó ausencia de al menos uno de sus dientes y del total de éstos, el $29.4 \%$ utilizaba prótesis dentales. La clasificación de Kennedy maxilar más prevalente fue Clase 3 y en la arcada Mandibular fueron similares resultados para Clase 1 y 3.

Conclusión: Estos resultados demuestran la alta prevalencia de sujetos edéntulos en los distintos servicios de salud de las islas y la necesidad de realizar programas de promoción y prevención para minimizar el daño en la salud de las personas de estas localidades.

Palabras clave: edentulismo, prevalencia, Quemchi.

\footnotetext{
* Fuente de apoyo económico: Departamento de Salud, Ilustre Municipalidad de Quemchi. Presentación parcial de los resultados: Un avance de este trabajo fue presentado en las XXXI Jornadas de Salud Pública organizadas por la Escuela de Salud Pública Dr. Salvador Allende G. de la Universidad de Chile.
} 


\section{ABSTRACT}

Aim: To determine the prevalence of edentulism and Kennedy topographic classification in the population aged over 20 years of the Butachanques and Tac islands, Quemchi, Chile.

Materials and Methods: Descriptive study. We included subjects aged over 20 years at health services of the Butachanques and Tac islands during the months of August and September 2013. Subjects were asked to sign an informed consent form. The information collected was entered into a data entry form. The variables used were gender, age, number of teeth present, and type of prosthesis and edentulous type according to Kennedy's topographic classification. Descriptive statistics were determined using SPSS 18 (IBM® SPSS® software) statistical software.

Results: 88 subjects, $39.7 \%$ men and $60.3 \%$ women, were evaluated. $89.7 \%$ of the subjects had absence of at least one of their teeth and $29.4 \%$ of these were using dentures. The most prevalent maxillary classification was Class 3, while the mandibular arch had similar results for class 1 and 3.

Conclusion: These results demonstrate a high prevalence of edentulous subjects in the various bealth services of the islands, and the need for promotion and prevention programs to minimize damage to the bealth of people in these localities.

Keywords: edentulism, prevalence, Quemchi.

\section{INTRODUCCIÓN}

El edentulismo parcial y total corresponde a la ausencia de algunos o todos los dientes. Las personas que presentan esta condición ven afectada su calidad de vida al presentar limitaciones al hablar, comer y sonreír, esto último debido al compromiso estético que provoca no tener todos los dientes. ${ }^{1}$ Las causas de pérdida dentaria son variadas, siendo las principales: caries y enfermedad periodontal. ${ }^{2}$ Ambas enfermedades son prevenibles y es posible evitar la pérdida de dientes siempre que exista el acceso a atención odontológica en una etapa en que la enfermedad no esté avanzada y se pueda realizar un tratamiento conservador.

En la población chilena, la prevalencia de edentulismo en el año 2003, para el rango de 35-44 años fue de 79,7\% (solo edentulismo parcial) y de 98,9\% para el rango de 65-74 años. De este último grupo, un $29,1 \%$ presentó edentulismo total. ${ }^{3}$

Entre los factores de riesgo de aumento de la indicación de exodoncia como tratamiento definitivo encontramos: difícil acceso a la atención odontológica, ${ }^{4}$ pobre higiene oral, ${ }^{2,4}$ bajo nivel educacional, ${ }^{4}$ vivir en sector rural, ${ }^{4}$ y la edad, este último factor considerado por las condiciones especiales de cuidado y enfermedades prevalentes a medida que aumentan los años. ${ }^{2,1}$

Dentro de la comuna de Quemchi, ubicada en la Isla Grande de Chiloé (región de los Lagos, Chile), se encuentran las islas $\mathrm{Bu}$ tachauques y Tac, sectores donde hay un bajo acceso a la atención dental, y en los cuales no se puede realizar un tratamiento completo e integral a los sujetos dadas las condiciones de clínicas y de acceso para ellos y los profesionales. ${ }^{5}$ Para planificar futuros programas promocionales y preventivos es necesario conocer el estado bucodental de la población, ya que nunca se han recopilado estos datos en las islas mencionadas.

El objetivo de esta investigación fue determinar la prevalencia de edentulismo en la población mayor de 20 años que acudiera a atención bucodental de los distintos establecimientos de salud de las islas Butachauques y Tac, durante el periodo de agosto a septiembre del año 2013. 


\section{MATERIALES Y MÉTODOS}

Diseño de Estudio. Estudio Observacional Descriptivo de corte transversal, aprobado por parte del comité de ética del Centro de Salud Familiar (CESFAM) de Quemchi.

Contexto. La investigación y recolección de datos se llevó cabo en el Centro Comunitario de Salud Familiar (CECOSF) de Metahue, Posta de Aulín, San José, Coneb, Maluco, Nayahue y Tac; durante los meses de agosto y septiembre del año 2013. La evaluación clínica fue realizada por un estudiante Interno y el registro fue llevado a cabo por otro estudiante.

Características. Las islas nombradas presen$\tan 100 \%$ de población rural. Cada sector cuenta con un establecimiento de salud, siendo el mayor el CECOSF del sector Metahue, el cual posee un box dental equipado con sillón dental. Los demás sectores presentan postas de salud rural no equipadas para atención bucodental. El agua de la población insular no es potable ni fluorada.

Atención bucodental. Cada dos meses, el equipo odontológico del CESFAM de Quemchi visita el CECOSF de Metahue y por una semana realiza acciones clínicas en el lugar. Para la isla Tac, todos los meses acude por un día el buque PMD (Patrullero Médico Dental) Cirujano Videla a realizar atención clínica, centrándose en urgencias.

\section{Participantes}

Población objetivo fue toda persona mayor de 20 años; población elegible fueron aquellos sujetos de la edad estipulada que asistieron a atención bucodental en los distintos establecimientos de salud de las islas $\mathrm{Bu}$ tachauques y Tac durante los meses de agosto y septiembre.

\section{Criterios de inclusión/exclusión}

Los criterios de inclusión fueron: personas mayores de 20 años que asistieran a atención odontológica en los distintos establecimientos de salud pertenecientes a islas Butachauques y Tac durante los meses de agosto y septiembre; estar inscritos en las fichas familiares del CECOSF de Metahue y posta rural de isla Tac; firmar consentimiento informado que indique su participación en el estudio.

Los criterios de exclusión fueron: no cumplir los criterios de inclusión; sujetos que asistieran de atención de urgencia; sujetos que no permitiesen su revisión bucodental por mala conducta, cambios bruscos de personalidad o que no se encuentren en condiciones óptimas cognitivas el día de la evaluación.

\section{Variables}

Se confeccionó una ficha correspondiente al estudio, que incluyó las siguientes variables:

Género: se registró como masculino o femenino.

Edad: se registró la edad en años, sin considerar meses ni días.

Portador de prótesis removible: se registró si el sujeto era portador o no de cualquier tipo de prótesis removibles, ya sean parciales acrílicas o metal acrílica; o completas acrílicas. No se midió la calidad de las prótesis removibles utilizadas por los sujetos. Número de dientes presentes: se registró el número de dientes que el sujeto tuviera en boca, excluyendo aquellos dientes que presentaron enfermedad periodontal severa con movilidad, dientes con indicación de extracción, dientes con mal pronóstico rehabilitador y terceros molares. Si algún sujeto presentó en una arcada solo terceros molares, fue registrado como desdentado total. Se consideró así, ya que en la evaluación los terceros molares presentes presentaron indicación de exodoncia.

Clasificación de Kennedy: Para la clasificación de las arcadas parcialmente dentadas, se utilizó la clasificación de Kennedy, la que categoriza las arcadas en distintas clases. Además se incluyeron dos categorías más, la de Desdentado y Dentado total, que no son incluidas dentro de la clasificación de Kennedy original:

- Clase 1: áreas edéntulas bilaterales posteriores a los dientes naturales.

- Clase 2: área edéntula unilateral posterior a los dientes naturales remanentes. 
- Clase 3: área edéntula unilateral con dientes naturales remanentes delante y detrás del área edéntula.

- Clase 4: área edéntula única bilateral (que atraviesa la línea media), anterior a los dientes naturales remanentes.

- Desdentado total: se incluyó esta clasificación cuando se registró un sujeto que no presentase ningún diente en la arcada evaluada.

- Dentado total: consideramos sujetos que presenten 28 dientes en boca, excluyendo los terceros molares.

\section{Selección de la muestra}

Se consideró por conveniencia, el universo completo de sujetos mayores de 20 años que consultasen en los distintos centros de salud de las islas Butachauques y Tac. Se utilizó este tipo de selección ya que no existen registros de la población total de las islas.

\section{Unidad de estudio}

La unidad de estudio fueron las arcadas dentarias de sujetos mayores de 20 años.

\section{Consentimiento informado}

Se diseñó un consentimiento informado, el cual fue previamente aprobado por el comité de ética del CESFAM de Quemchi. En caso de que el sujeto se negase a firmar, fue excluido del estudio.

\section{Métodos estadísticos}

Los datos recopilados fueron ingresados a una planilla Google Doc, en la cual se categorizaron las variables en números enteros, para luego realizarse un análisis de estadística descriptiva mediante SPSS 18 (IBM® SPSS ${ }^{\circledR}$ software).

\section{RESULTADOS}

Durante los meses de agosto y septiembre de 2013, dos internos de Odontología previamente capacitados, realizaron la evaluación de 88 sujetos entre el rango etario de 20 a 75 años en los distintos centros de salud de las islas Butachauques y Tac. Respecto a las características del grupo evaluado, el 39,7\% correspondió a hombres, $60.3 \%$ a mujeres. Del total de evaluados un $89,7 \%(\mathrm{~N}=70)$ de los sujetos presentó ausencia de al menos uno de sus dientes y, de estos, el $29,4 \%$ $(\mathrm{N}=26)$ utilizaba prótesis dentales. La Tabla 1 presenta los valores encontrados por rango etario y su promedio de dientes en boca por grupo.

Se presentan los resultados encontrados para la Clasificación de Kennedy de ambas arcadas en la Tabla 2. Los resultados sobre la clasificación topográfica de Kennedy encontrados para la arcada maxilar muestran mayor porcentaje de Clase 3 (42\%) y para arcada mandibular un similar porcentaje para Clase 1 y $3(29 \%)$, siendo la Clase 4 la menos prevalente para ambas arcadas.

\section{DISCUSIÓN}

En concordancia a lo reportado en el presente estudio, otra investigación realizada en la población Mapuche-Huilliche de la Isla Huapi, Chile, también encontró altos valores de edentulismo $(98,4 \%)$ y, de éste, un $12,5 \%$ de los sujetos evaluados poseía alguna arcada edéntula. Al igual que en el recién citado estudio, se considera que el entorno social (nivel socioeconómico, nivel educacional, dificultad de acceso a la atención odontológica) en que se encuentra esta población sería determinante para explicar la gran cantidad de daño encontrado. ${ }^{6}$

Debemos destacar el mayor porcentaje de mujeres $(60,3 \%)$ que acudieron a la evaluación bucodental, en comparación con el porcentaje de hombres $(39,7 \%)$. Lo anterior puede ser explicado debido a que las mujeres asistían a los centros de salud por llevar a sus hijos a controles y aprovechaban esta instancia de atención bucodental.

Dentro de las características de la población evaluada, se considera importante mencionar que el $29,4 \%$ de quienes presentaban ausencia de al menos un diente utilizaban algún tipo de prótesis parciales removibles, lo que se debe a que existen programas de entrega de este tipo de tratamiento para sujetos 
Tabla 1. Número de dientes presentes en boca por paciente, según rango etario.

\begin{tabular}{cccc}
\hline Rango Etario & Pacientes (n) & Promedio de dientes & Mín - Máx \\
\hline $21-34$ & 37 & 24 & $15-30$ \\
$35-44$ & 23 & 17 & $10-26$ \\
$45-54$ & 13 & 14 & $7-20$ \\
$55-64$ & 10 & 12 & $9-18$ \\
$65-74$ & 5 & 10 & $2-15$ \\
Todas las edades & 88 & 15 & \\
\hline
\end{tabular}

Tabla 2. Distribución de sujetos evaluados (\%) según presencia o ausencia de dientes en arcadas

\begin{tabular}{ccccccc}
\hline & $\begin{array}{c}\text { Dentado } \\
\text { total (\%) }\end{array}$ & $\begin{array}{c}\text { Desdentado } \\
\text { total (\%) }\end{array}$ & \multicolumn{3}{c}{$\begin{array}{c}\text { Desdentado parcial } \\
\text { según Clasificación de Kennedy }\end{array}$} \\
\hline & & & Clase 1 (\%) & Clase 2 (\%) & Clase 3 (\%) & Clase 4 $(\%)$ \\
Arcada maxilar & 19 & 6 & 16 & 10 & 42 & 7 \\
Arcada mandibular & 18 & 0 & 29 & 22 & 29 & 2 \\
\hline
\end{tabular}

de población insular, que cumplan con ciertos requisitos especificados en su ficha clínica y con previa evaluación de asistente social. A pesar de esto, se requiere que futuras investigaciones evalúen el estado de estas prótesis y la necesidad de este tratamiento por parte de la población.

Acerca de los resultados obtenidos para la Clasificación Topográfica de Kennedy, respecto al edentulismo, no se encontró literatura con resultados de este tipo para otra población chilena, ya sea urbana o rural, con el propósito de realizar comparaciones. Este tipo de estudios son necesarios en la población para generar nuevas líneas investigativas y presentar realidades antes no evaluadas. A nivel internacional, un estudio realizado por la Universidad Médica Nacional de Kazakstán evaluó a 112 sujetos, encontrando que la clasificación de Kennedy más prevalente fue Clase 3 para ambas arcadas $50 \%$ maxilar y $41,1 \%$ mandibular) y la menos prevalente fue Clase 4 (7,1\% maxilar y 5,6\% mandibular). ${ }^{7}$ Estudios recientes de otros países, (por ejemplo Jordania, Nigeria y Arabia Saudita) indican que la Clase 3 es el tipo de edentulismo parcial más prevalente. ${ }^{7,8}$
Limitaciones del estudio. Uno de los factores que más afectó la pesquisa de sujetos fue la dificultad para el acceso de estos a los distintos establecimientos de salud, así como el tiempo de que se dispuso para efectuar la investigación, como lo fue el desinterés de varios sujetos por realizar un control o revisión odontológica, ya que desvalorizan la oportunidad de detectar a tiempo alguna enfermedad y solo se motivan por atención en momentos en que presentan dolor o una lesión dental afecta gravemente su estética. Consideramos además que, para futuras investigaciones, es necesario obtener el registro real de la población, para así estimar un tamaño muestral representativo. En el caso del presente reporte, al no poseer este dato no fue posible realizar un cálculo del tamaño muestral, lo que se convierte en una limitación al momento de querer extrapolar los resultados a la población total.

Esta investigación responde a una necesidad de la comunidad de Quemchi y sus autoridades, de tener un acercamiento objetivo sobre el estado de salud bucal de las islas Butachauques y Tac. Se ha trabajado en conjunto, generando una alianza entre la Escuela de Odontología de 
la Universidad Austral de Chile y la Municipalidad, ${ }^{9}$ con la finalidad de trabajar en conjunto por mejorar la salud bucodental de la población más vulnerable. Lo anterior requiere de un trabajo planificado, objetivo y sistemático al que se deben seguir sumando otras investigaciones que permitan realizar acciones rehabilitadoras, de prevención y promoción de la salud con la finalidad de contribuir a mejorar la salud de esta población.

\section{AGRADECIMIENTOS}

Deseamos expresar nuestros agradecimientos a la Ilustre Municipalidad de Quemchi por permitirnos realizar atención clínica en su comuna, y al Departamento de Salud Quemchi por las gestiones realizadas para alojamiento y trabajo los meses señalados en las islas Butachauques e isla Tac.

De igual forma, a todos los funcionarios de la salud, personal de las instituciones educacionales rurales y familias que brindaron su apoyo.

\section{REFERENCIAS}

1. Carr A, Mcgivney G, Brown D. McCracken. Prótesis Parcial Removible. $11^{\mathrm{a}}$ ed. España: Elsevier; 2006.

2. Arteaga O, Urzúa I, Espinoza I, Muñoz A, Mendoza C. Prevalencia de caries y pérdida de dientes en población de 65 a 74 años de
Santiago, Chile. Rev Clin Periodoncia Implantol. Rebábil. Oral. 2009; 2(3): 161-66.

3. Chile. Ministerio de Salud. Diagnóstico de situación de salud bucal Chile 2010: análisis de situación salud bucal. Chile: MINSAL; 2010.

4. Belaude M. Relación entre el acceso a la atención dental y el edentulismo en pobladores de 18 a 65 años de edad del distrito de Cajamba, provincia de Cajamba, departamento de Cajamarca, durante el periodo de febrero - mayo de 2011 [tesis]. Perú: Universidad Peruana Cayetano Heredia; 2011.

5. Chile. Ministerio de Salud. Diagnóstico de Salud, Comuna de Quemchi. Chile: MINSAL; 2011.

6. Angel P. Prevalencia de caries, pérdida de dientes y necesidad de tratamiento en población adulta Mapuche-Huilliche de Isla Huapi. Rev Clin Periodoncia Implantol. Rehabil. Oral. 2010; 3(2): 69-72.

7. Al-Dwari Z. Partial edentulism and removable denture construction: a frequency study in Jordanians. Eur J Prosthodont Restor Dent. 2006 Mar; 14(1): 13-7.

8. Sadig W, Idown A. Removable partial denture design: a study of a selected population in Saudi Arabia. J Contemp Dent Pract. 2002; 15: 40-53.

9. Lehmann P. Estudiantes de Odontología culminan su carrera con servicio a la comunidad. Facultad Al Día [en línea]. 2013 Nov 29 [consultado 29 abril 2014]. Disponible desde: http://www.medicina.uach.cl/noticia.php?id=1744 